\title{
An Infinite-Light and Infinite-Frequency in Cosmology and Neurosciences
}

\author{
Zamzuri Idris \\ Department of Neurosciences, School of Medical Sciences, Universiti Sains Malaysia, Kelantan, Malaysia \\ Email: neuroscienceszamzuri@yahoo.com
}

How to cite this paper: Idris, Z. (2019). An Infinite-Light and Infinite-Frequency in Cosmology and Neurosciences. Open Journal of Philosophy, 9, 236-251.

https://doi.org/10.4236/ojpp.2019.92016

Received: April 30, 2019

Accepted: May 21, 2019

Published: May 24, 2019

Copyright (c) 2019 by author(s) and Scientific Research Publishing Inc. This work is licensed under the Creative Commons Attribution International License (CC BY 4.0).

http://creativecommons.org/licenses/by/4.0/

\begin{abstract}
The aim of this paper is to introduce the new concept of an infinite-light and infinite-frequency. Research methodology includes literature review, new hypotheses and scientific explanations to support it are stated and clarified, and finally, suggestions are proposed on how to study it. In brief, this paper discusses a widely accepted big bang theory on creation of our universe which mentions formation of glowing or light before particle-formation phase, and concept of duality for an atom and light in relation to an infinite-light. These two notions clearly brought out light as a fundamental subject to discuss. In this manuscript, I hypothesize an existence of an infinite-light which has several peculiar features: 1) infinite-energy, 2) infinite frequency (waveless energy), 3) infinite time (timeless), and 4) infinite-space (infinite-universe). In connection with these features, an infinite-light can be theorized to exist either immediately after the big bang or prior to big bang. Interestingly, its existence can explain creation of the universe in further details. It might be able to unravel what has already existed before the big bang, what drives the isotropic and homogenous universe expansion, why there is cosmological constant and finally it may clarify matters related to consciousness, invisibility, black hole and dark energy. Nonetheless, one must bear in mind that most written in this manuscript are hypothetical and remain to be proven.
\end{abstract}

\section{Keywords}

Light, Big Bang, Consciousness, Infinite-Light, Infinite-Frequency, Universe Expansion, Infinite-Universe, Finite Universe, Vision, Near Death Experience, Brainwaves, Cosmos, Soul

\section{Introduction}

Cosmology and Neurosciences are two major and intricate subjects which attract many to study them. These two subjects are considered by some as inter-related. 
The outer cosmos (physical universe) gives information to the brain for further processing, and the processes that happen in inner cosmos (brain) are closely related to a concept of mind or consciousness (Arthur, 2019; Di Biase, 2009; Subhash, 2009). In relation to this argument, the element which may have strong connection between the outer and inner cosmos is the waves. Feature of a wave is an up and down or frequency or energy. As a consequence, cosmologist and neuroscientist commonly state the inner and outer cosmos are linked through frequency or energy, or they simply view that our universe is covered by frequency or energy, and thus one may conclude the whole thing as one or single consciousness (Doesburg, Green, McDonald, \& Ward, 2009; Keppler, 2018; Meijer \& Geesink, 2019). Therefore, quantum physics with emphasis on waves and light should be used in studying them (Figure 1). The obvious link noted here is a chain concept of particles-waves-light. It is mainly because of the brain or cosmos existence which can be viewed as either in combination of particles, waves and light form, or solely in waves form, or entirely in light form.

In pertaining to aforementioned notion, scientists have already known that an atom can either be particles or waves, similarly light can also be either particles or waves (Sakai, 2018; Yuan et al., 2018; Thomson, 1927; Arndt, Nairz, Vos-Andreae, Keller, Van Der Zouw, \& Zeilinger, 1999; Bohr, 1928; Compton, 1923; Du \& Zhao, 2010). Thus one may view that all are waves or all are originated from the light. Scientists have also theorized that our universe was started from a big explosion or better known as big bang with a cascade of

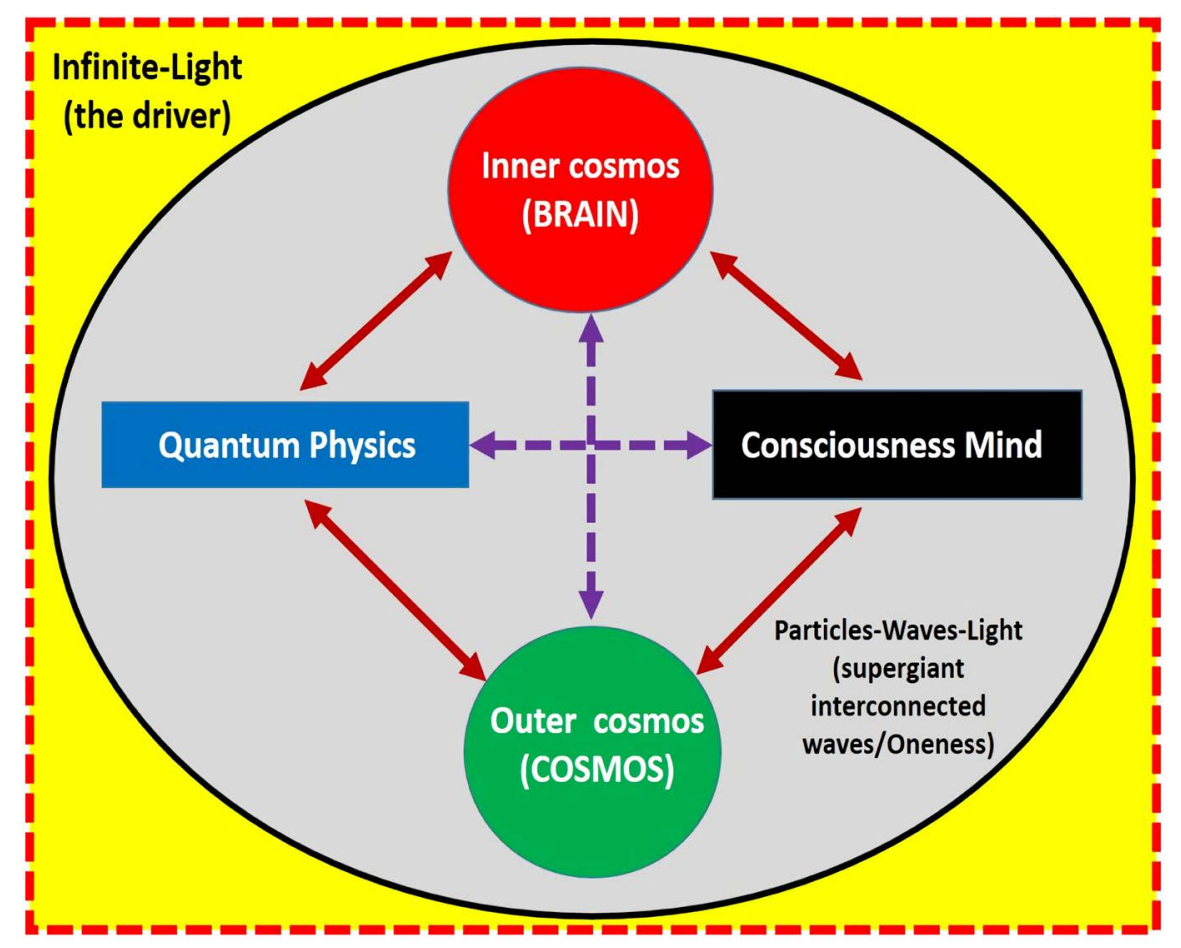

Figure 1. The relationship between outer and inner cosmos. Waves and light may play a vital role in their relationship, and an infinite-light is theorised as an element that gives rise to all (unification theory). 
glowing-waves-particles-atoms-molecules-matter-and many objects formations (Hubble, 1927, 1929; Hawking \& Ellis, 1973; Vilenkin, 1983; Guth \& Kaiser, 2005). In relation to this acceptable theory of creation, space-time is also emphasized. When considering these two aspects of science (particles-waves-light for all in the universe, and big bang for creation), one may conclude that light is fundamental in our life or creation. Therefore, in this hypothetical manuscript, I theorize that a non-ordinary infinite-light does exist and it contributes a lot in our universe formation, in our life, in our universe expansion and in conservation of energy. In other words, one may say "light itself is originated from the light (higher light)!" Finally, this manuscript also correlates this infinite-light or infinite-energy with single or one consciousness, invisibility and black hole.

\section{The Hypotheses: Creation of Physical Universe Is Related to Either an Infinite-Light Existing Immediately after the Explosion Or an Infinite-Light Existing before the Implosion-Explosion}

The origin of our universe has been debated for a long time. At this current moment, nobody can confidently explain how our universe came into existence. The Big-Bang Theory is only a theory but has received much attention from scientists. The theory explains the existence of our universe as resulting from a big explosion that occurred long time ago (Hubble, 1927, 1929; Hawking \& Ellis, 1973). However, the big bang model as a viable explanation for the origin of the universe has not been accepted by some cosmologists. The singularity concept that contained infinite density and energy that forms a basis for a big bang model has been questioned by many scientists (Arp, Burbidge, Hoyle, Narlikar, \& Wickramasinghe, 1990; Van Flandern, 2002; Ahmed \& Saurya, 2015; Ratcliffe, 2010). At present, the questions that are related to why this singularity existed, how it is originated, why it exploded and why the universe expands in isotropic and homogenous manner cannot be satisfactorily explained. Ratcliffe (2010) also questioned the validity in interpreting the red shifts as supporting a big bang model. Thus, this paper try to address these issues by arguing a true presence of an infinite light. Thus, my hypotheses related to these question are an infinite-light which is not similar to ordinary light, has been existed either before or immediately after the big bang. In other words, the creation of physical universe is related closely to this infinite-invisible-light.

In the context of an infinite-light existing immediately after the big bang or explosion, it is important to appreciate that this infinite-light is not like an ordinary light. This infinite-light is viewed as a non-particle light, which existed before the wave- or particle-formation phase. Because of its infinite feature, it is able to spread and covers isotropically and homogenously the infinite-space. Subsequently, tiny portion of this early tremendous light gets converted to ordinary or photonic light (lower light), waves, particles, atoms and other objects which form a wonderful life or physical universe. Thus, we may view that the 
physical universe expansion is driven by this invisible, still present and undetectable infinite-light. In other words, an infinite-light is an invisible background or fabric for physical universe expansion.

In reference to second hypothesis where an infinite-light had already existed before the big bang, the formation of physical universe was not via explosion alone, it might involve both implosion and explosion or easily stated as implosion-explosion. A tiny portion of a tremendous infinite-light which covered an infinite-space suddenly became suck-inward and then exploded with a giant bang (if there were multiple finite-universes, their formations were also likely in this fashion, that is: tiny portion of an infinite-light implosion-explosion). This initial bang had resulted first in formation of non-infinite or finite-light, then waves, particles, atoms, matters and so on to form a wonderful physical universe (life). This physical universe expands isotropically and homogenously because of a fabric provided by an infinite-light. Thus, one may say prior to big bang, there was, and still an infinite-light existing now. This second view is preferred because its existence happened before existence of time (big bang) — see discussion on infinite-frequency later. Figure 2 shows these two possible hypotheses or theories.

\subsection{What Is an Infinite-Light?}

We already knew that ordinary light can either be particles or waves. Similarly, we also knew that an atom can also be either particles or waves. We might thus say that an apparent atom is also light or originated from the light. This is simply because of the light-waves-particles cascade (the origin) that existed after the big bang. Therefore, human or other objects actually can be viewed in three main forms: 1) in physical form (particles-atoms); 2) in wave form [deeper view]; and

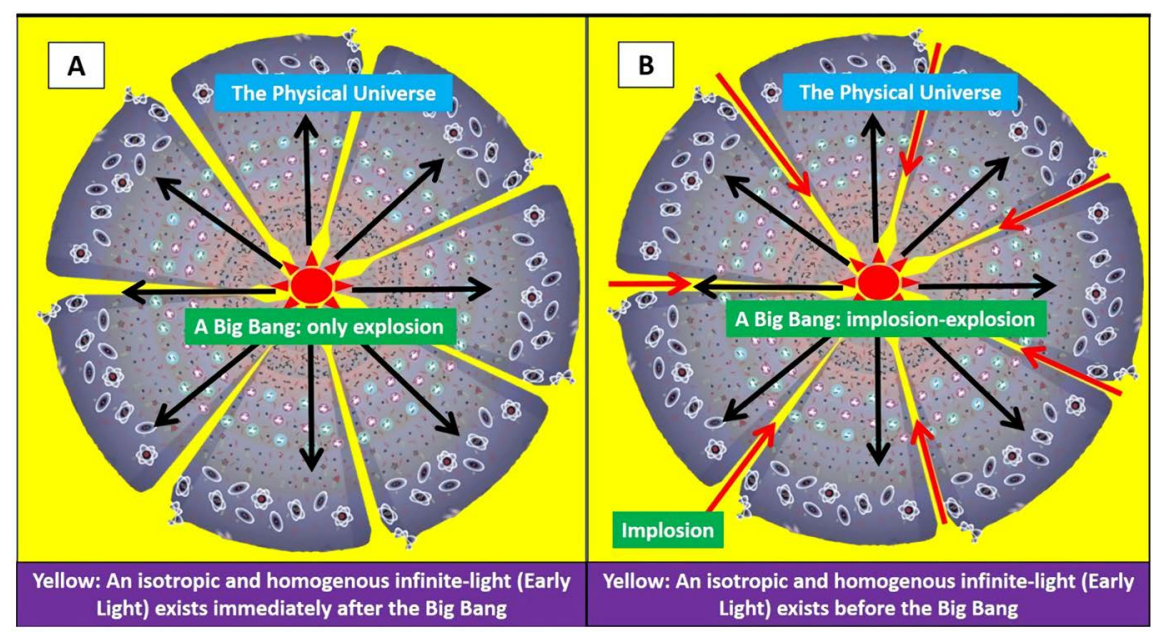

Figure 2. Two theories of physical universe formation. Theory A: The physical universe was formed from a big bang that resulted in formation of an infinite-light immediately after the explosion. Theory B: The physical universe was formed from a tiny portion of homogenous infinite-light which retracted to a single dot (implosion) prior to a massive big bang (explosion). Isotropic and homogenous expansion of a newly created physical universe follows the fabric provided by an infinite-light with conservation of a single and an infinite-energy via transitions (i.e. one consciousness). 
3) in light form [the deepest view] (Idris, 2014; Idris, Muzaimi, Ghani, Idris, Kandasamy, \& Abdullah, 2014). This thought may conclude that all is either fundamentally waves or light or in other words, all are originated from the light. Deeper thought on this matter brings out the conclusion that any light in physical universe (finite-light) was also originated from this early light (infinite-light)! This can be made known as light upon light. This initial light (even ordinary light is originated from it) is named here as an infinite-light.

This infinite-light is hypothesized as the first created item and currently still covering whole of the infinite-universe (metaphysical universe). It covers whole of universe simply because it is infinite in its energy and it is invisible simply because it is waveless light and not related to time (an infinite light existing prior to big bang or prior to beginning of time, as stated in the second hypothesis). Thus one may conclude that this infinite-light is a metaphysical light, forming a metaphysical universe. In order to understand its properties, let's us look at property of a wave. A wave can merely be defined as ups and downs or an energy. It happens when there is time - as previously described in section of physical universe formation or light-waves-particles cascade. Thus, ordinary light is actually a wave or wavy item and exists when there is time (space or physical universe). When there was no time existing, the light which is a major subject of discussion can no longer be regarded as an ordinary light; instead, it should be interpreted as an infinite-light or tremendous light or metaphysical light. On that account, an infinite-light is waveless (having infinite-frequency or infinite waviness) which consists of "all frequencies and has infinite-energy"! Therefore, an infinite-light covers whole of universe (infinite-space) and it is invisible to the eyes! Figure 3 explains its characteristics.

\subsection{Invisible to the Eyes (Brain), Dark Energy and Spirit}

An infinite-light is invisible to the eyes because it is waveless. All inside physical universe is thought as wavy and hence can be detected, studied and/or seen. To sum up, we are capable in perceiving an object because of: 1) presence of the light; 2) the light is reflected; and 3) object is wavy. Thus, the visual cortices (part of the brain) can detect or see most of the wavy objects (brain and visible light spectrum). This might occur through coherence wave signals that pass directly to the brain (remember all can be viewed as waves) or through quantum entanglement which forms a faster interaction. Nevertheless, this type of communication is still under quantum-investigation (Georgiev \& Glazebrook, 2018; Zarkeshian, Kumar, Tuszynski, Barclay, \& Simon, 2018; Shi, Galvez, \& Alfano, 2016; Cocchi, Gabrielli, Tonello, \& Tuszynski, 2017). This invisible- and infinite-light which has infinite-energy and may cover an infinite-space might form a driving force for an isotropic and homogenous physical universe expansion. In other words, it is a fabric or background for physical universe expansion or might consider it as dark energy! Detailed thought on this concept, one may view that a visible-physical universe is in fact lying inside the invisible-metaphysical universe. Thus, there could be some transitions of item happen between them. 


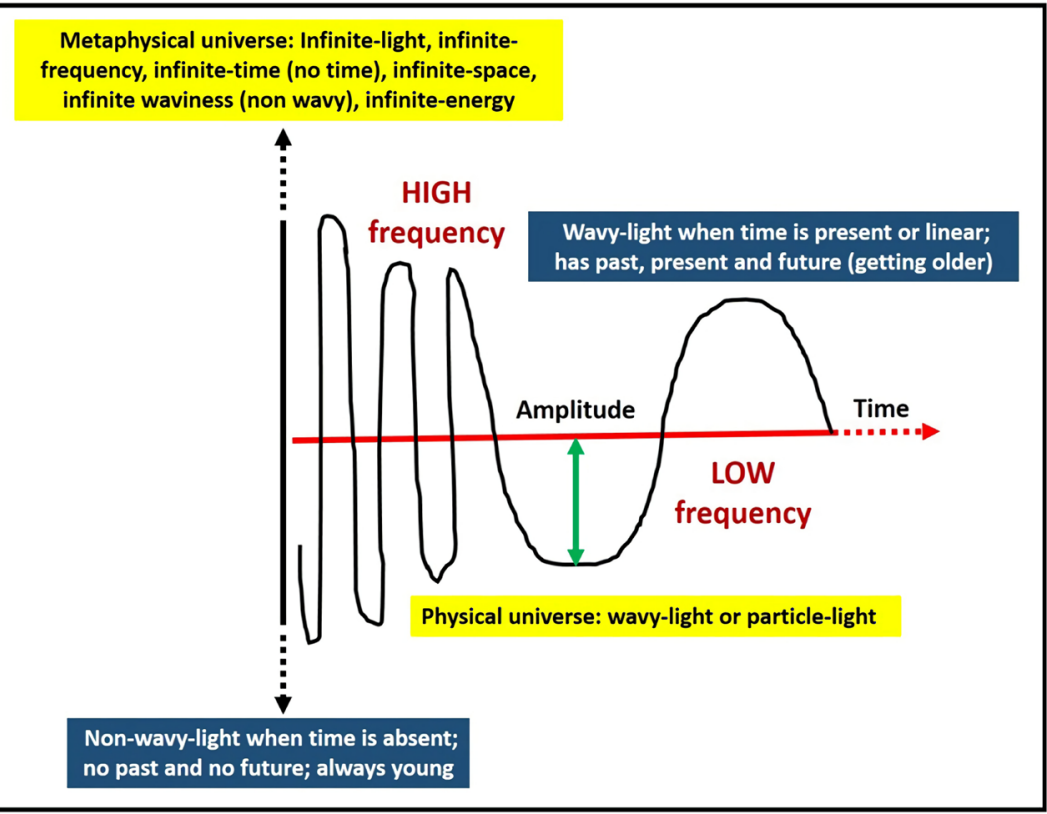

Figure 3. Depicting high and low frequency waves. A higher-frequency wave has more energy than a lower-frequency wave with the same amplitude; and a higher-amplitude wave has more energy than a lower-amplitude wave with the same frequency. Therefore, an infinite-light with infinite-frequency or infinite-energy is waveless, and it is capable in yielding all finite-frequency range (originated from it); in other words, "frequency shift" is the key in formation of physical universe and all inside it.

Could this be the light or soul or spirit?-that is being born (appear or exist): appear or in finite form via "frequency shift", that is from infinite-frequency to lower-frequency, and being dead: disappear or revert to an infinite form, that is from low-frequency back to infinite-frequency (Figures 4(A)-(D)). For this particular point, I do not want to go into further details and leaving it for readers to think themselves. Figure 4 depicts the existence of two universes - visible and invisible or finite and infinite universes or physical and metaphysical universes; physical universe expansion; and the transitions that might exist between them. Perhaps, worthy to note here that, the transitional material must at least possess an infinite-light like feature, simply because we talk about an interaction between physical and metaphysical universe (an infinite-light). For this reason, the transitional material is hypothesized as spirit (when it reverts to its own world of abstract entity) or soul (when it governs our body) or mind (when we refer it as act of thinking or cognition) or heart (when it received intuitive illumination) [note: different names might finally refer to same or one name i.e. the spirit] (Figure 4(A) and Figure 4(C)).

\section{Isotropic and Homogenous Physical Universe Expansion, Conservation of a Single Infinite-Energy or Infinite-Light, One Consciousness, and Albert Einstein with an Infinite-Light}

Afshordi and Magueijo came up with their theory to explain why the cosmos 


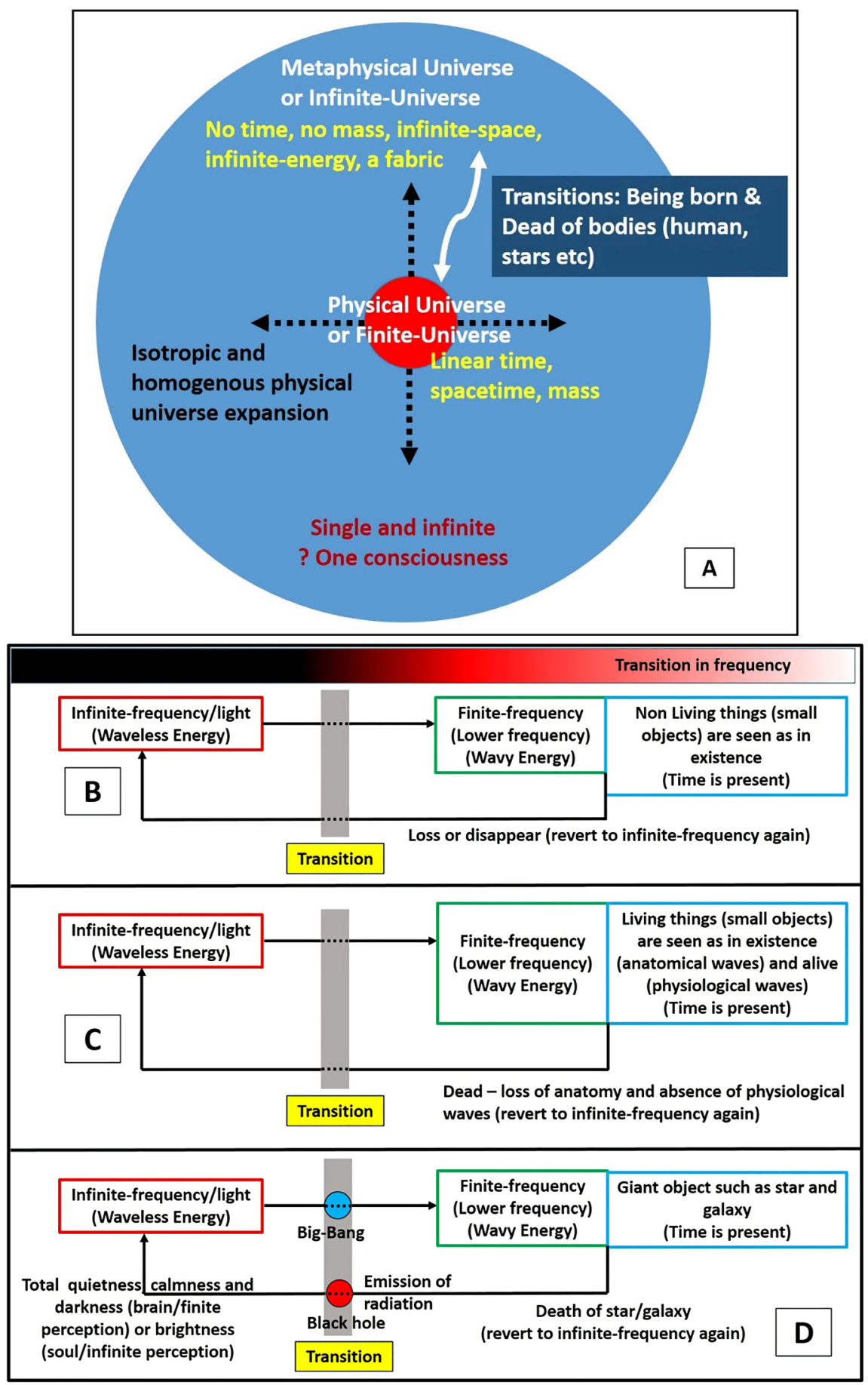

Figure 4. A: Depicting a conservation of a single and yet infinite energy, the transition between infinite and finite universes, one whole consciousness and isotropic and homogenous universe expansion based on the fabric provided by an infinite-light which might unify all frequencies or theories. B, C and D: Transition in frequency for "small and giant objects". The smaller the object/particle, the larger the oscillation/wave (energy).

looks much the same over vast distances [isotropy and homogeny] (Afshordi \& Magueijo, 2016). To be so uniform, light rays must have reached every corner of the cosmos, otherwise some regions would be cooler and denser than others. 
According to their calculations, the heat of universe in its first moments was so intense that light and others moved at infinite speed. Under these conditions, light reached the most distant pockets of the universe and made it look as uniform as we see it today. This interesting theory views light as an essence that has an "infinite" speed at the birth of the universe when the temperature of the cosmos was a staggering ten thousand trillion trillion celsius. This interesting light concept at the early beginning of the universe (immediately after the big bang) seems to suit my first aforementioned hypothesis in this manuscript. However, this hypothesis or theory seems inevitably incorporating time as its essence. Noteworthy, immediately after the big bang, there was already time existing; in consequence, it is against my descriptive feature of an infinite light which is waveless because its existence is not related to time at all. Nonetheless, there is still a possibility that it started to appear immediately after the big bang (let's say after $10^{-37} \mathrm{~ms}$ !), but theoretically it is not infinite in its feature! (only massive or nearly infinite-energy). This background light still has a potential to become a steering force for an isotropic and homogenous physical universe expansion. On the other hand, my second hypothesis which stated an infinite-light had already existed before the big bang seems more plausible. This is mainly because its initial existence which unrelated to time, and followed by a big bang (implosion-explosion) enable one explaining its infinite or metaphysical (infinite universe) or invisible feature. Its ability to remain in existence even after a big bang might denote that it can be a steering invisible force for a physical universe expansion in isotropic and homogenous manners (remember it is infinite-energy, and might only a tiny portion of its energy is used-up in formation of our or one physical universe and theoretically, multiple universes can be originated from it).

Figure 4(A) clearly shows a homogenous and isotropic physical universe expansion. This expansion might arise because of two phenomena: 1) an equally distributed background infinite-energy (or infinite-light); and 2) transitions that happen between these two universes (energy transition). Thus, one may view that these two universes are actually a single and yet infinite in its feature (seen as two but they cannot be separated) or one may say "there is conservation of a single and infinite-energy" (singularity: singular with infinite values) or presence of a cosmological constant (Einstein, 1917; Barrow \& Shaw, 2011). There is no loss of energy or infinite-energy is always preserved! As a result of this concept, one may also view it as one or single consciousness which gives tremendous and endless energy to both universes! (Meijer \& Raggett, 2014). This concept seems in agreement with another life-perspective which views all as waves, including brain, table, animals etc. and hence one may define consciousness as one or single - simply because all in physical universe can form a supergiant interconnected waves which has coherence and incoherence wave interactions; and this supergiant network of waves (finite-energy/wavy energy) lies inside waveless energy (infinite-invisible-energy with total quietness and calmness). Therefore, one may also say both universes are indeed one! In subsequent subtitle, I am 
going to explain an $\mathrm{E}=\mathrm{MC}^{2}$ formula which made famous by Albert Einstein, is also capable in explaining the existence of a single and yet infinite-energy at the beginning or before the creation.

\subsection{Albert Einstein and an Infinite-Light}

The two aforementioned theories of a single and yet infinite-light (an infinite-light appeared immediately after the big bang; and an infinite-light appeared prior to big bang) suit the philosophical idea of a dot or single-concept for physical universe origin (singularity with infinite-values). The famous $\mathrm{E}=$ $\mathrm{MC}^{2}$ formula made famous by Albert Einstein could also demonstrate the existence of an infinite-light prior to big bang (supporting the second hypothesis). Let's consider these two early situations:

\subsection{Before Formation of Physical Universe}

I) At time equals to zero (timeless or non-existence), the dimension (distance) was also zero or in non-existence, then the value of $\mathrm{c}$ is everything (zero divided with zero is everything; note that $c=$ distance or dimension divided with time, hence $c=0 / 0 ; c \times 0=0 ; c$ is everything). Therefore, at time is zero (timeless), everything can either be in a concentrated form at "one point or one dot" or "everything is dispersed equally and infinitely" that is as "a single and yet infinite". Pertaining to this, at time is zero, hence mass would also be zero (since no time is existing yet), then the energy concept for "physical life" would also be zero $(\mathrm{E}=0 \times$ everything $=0$ ) or in other words, there is no physical life (finite-energized objects) yet.

II) When time is zero and dimension is not zero (got values), then the value of $c$ is infinity (number is divided by zero). At this zero time but dimension is not zero, the energy is also zero. It is simply because at non-existence of time, the mass is also not in existence $(\mathrm{E}=0 \times$ infinity $=0)$. We can frankly infer three things here: 1) at zero time but presence of unknown or infinite (too big) dimension (could this be a single and yet infinite-light?), and the energy concept for "physical life" would still be zero or absence; 2) if there were extra unknown dimensions existing, they are unlikely related to time and have no mass (invisible or metaphysical dimension; again might denote an infinite-light or metaphysical universe); and 3) everything is infinity or infinity is everything: $\mathrm{E}=0 \times$ everything $=0$ for first circumstance, and $\mathrm{E}=0 \times$ infinity $=0$ for subsequent circumstance (infinity covers everything; infinite-frequency does cover or gives rise to all frequencies "via frequency shift").

The summary for point I) and II) above is: at timeless and dimension is either nonexistence or existence (metaphysical, too big, infinite or unknown dimension), then the value of $\mathrm{c}$ is either "everything" or "infinity" (note that: infinity is everything and everything is infinity). Thus "c" could be "an infinite-light" (infinity) or "a light of everything" which surpasses time, defying gravity and could be a source for all creations (everything)! Noteworthy, "c" is originally referred 
to the speed of light; and at zero time, this light i.e. " $c$ " is not an ordinary light but instead an infinite-light at infinite-speed! In other words, at infinite-speed, the time is zero or simply stop or not in existence due to time dilation concept. Therefore, one may conclude that the first creation is this infinite-light (infinitely fast and massive and no age) (see Figure 2).

\subsection{Formation of Physical Universe}

When time exists, let's say $10^{-37} \mathrm{sec}$ (the smallest number), the " $\mathrm{c}$ " value now becomes so massive (the biggest number, i.e. not infinite anymore - it is obtained when divided with the smallest number). Existence of time would cause subsequent existence of mass (physical item, particles etc.). Based upon the $\mathrm{E}=$ $\mathrm{MC}^{2}$ formula, the $\mathrm{E}$ value would be massive. This can be stated as follow: $\mathrm{E}=$ mass (existing) $\times$ massive value (time starts to appear) $=$ massive energy (near infinite value). Hence, the transition in energy value from none (time is absence) to massive (time starts to appear) would of course lead to a "massive or big explosion". From this massive explosion, better known as the Big-Bang, the time starts to progress; the glowing (according to the first hypothesis: the initial-immediate glowing is an infinite-light; whilst according to a second hypothesis, the glowing is a finite-light), the waves and the particles (including the ordinary photonic- or particle-light) and the masses start to appear. The physical universe is expanding further and hence life starts to exist. This appears in tally with aforementioned conservation of a single and yet infinite-energy with a shift in light-wave-particle concept (a tiny portion of infinite-light is converted to finite-light; and an infinite-light always remained in infinite-energy entity with presence of physical universe that resulted from a finite-light cascade).

In conclusion, this formula is capable in explaining both hypotheses with regard to an infinite-light (see Figure 2): it can either be already existed before the big bang as an equally dispersed "infinite-light" (preferred) or comes to existence immediately after the big bang. This infinite-light forms the fabric for physical universe expansion. When viewed in term of conservation of energy: the energy is always maintained; simply because of the existence of the fabric (infinite-light) and the transitions that happen between the two universes: from infinite-light to physical items such as ordinary or finite light, particles, matters etc. Anyway, all are viewed as originated from the light! - that is from metaphysical to physical life or in other words, be appearing/being born, and from physical life back to metaphysical life, be disappearing/being dead. It means all are in here (only in different dimension or frequency) and the waves or energy is always kept constant or similar as an infinite-energy (i.e. single and infinite, or one consciousness). Our view is just an extension of a previously known and accepted concept on origin of our universe; and hence this new idea or theory which incorporating non ordinary and yet tremendous infinite-light can still be called as Big-Bang or an "Infinite-light Big-Bang" Theory. Before putting an end to this interesting discussion on two hypotheses or theories which related to an 
infinite-light, I would like to briefly discuss on topic related to Olber's paradox, light upon light, black hole and how to prove the existence of an infinite-light.

\section{Olber's Paradox, Finite- and Infinite-Light (Finite- and Infinite Universe), Light upon Light and Black Hole}

Olber's paradox is a paradox because of the apparent contradiction between our expectation that the night sky would be bright and yet our experience that it is black. The argument for be bright is the universe is full of stars, why does not the light from all of them add up to make the sky bright? Many different explanations or theories have been put forward to resolve this paradox (Poe, 1848). In relation to this, two major points to consider are our brain perception and the light. We perceive object because of our brain ability to detect the visible spectrum of electromagnetic waves or energy. Our brains are not seeing the light itself or the object itself, instead our brains are "detecting" the visible spectrum of the light so that "a copy of the object" is perceived. Naturally, during night time with total darkness, there is absent of light, hence our brains are unable to detect the visible light spectrum to perceive. Thus, what our brains perceive is darkness. Thus, two main reasons of darkness perception are: 1) total absorption of the light by the objects, or 2) total absence of the light. In the context of Olber's paradox, we perceive darkness rather than bright sky because of the finite-physical universe (it is not infinite, still expanding and filled-up by finite-light that is visible and detectable ordinary photonic-light). Since it is finite-universe, we can perceive object as far away as the distance light can travel; the light from stars farther away than that has not yet had time to reach us (the brains) and so can't contribute to making the sky bright. Another compelling reason is that the sky may not be bright with the visible light of all the stars is mainly because when a source of light is moving away from us, the wavelength of that light is made longer (redshift-dissipate in its energy or less wavy). This means that the light from stars that are moving away from us (redshift or beyond: becoming less wavy or even nearly flattened out) will no longer be visible. Both telling that our physical universe is not infinite (still expanding) and based on aforementioned argument on existence of complete invisibility of "waveless" infinite-light (infinite-frequency), one may alternatively state Olber's paradox is due to dissipation in waviness for the light that travels from far away stars. Therefore, another or third reason for darkness perception or absence to perceive in neuroscience is "waveless or nonoscillating character of an element or object or item" (see Figure 5). Back to the points related to an initial discussion which stated all can be viewed as waves or all are originated from the light (light-wave-particle cascade), and hence can be perceived and studied. Therefore, anything that waveless (nonoscillating) cannot be perceived or detected! Likewise for an infinite-light which is waveless, imperceivable and yet has tremendous or infinite-energy (i.e. special feature of an early or infinite-light or tiniest or unseen item with massive energy). This background energy which is infinite, creates our metaphysical 


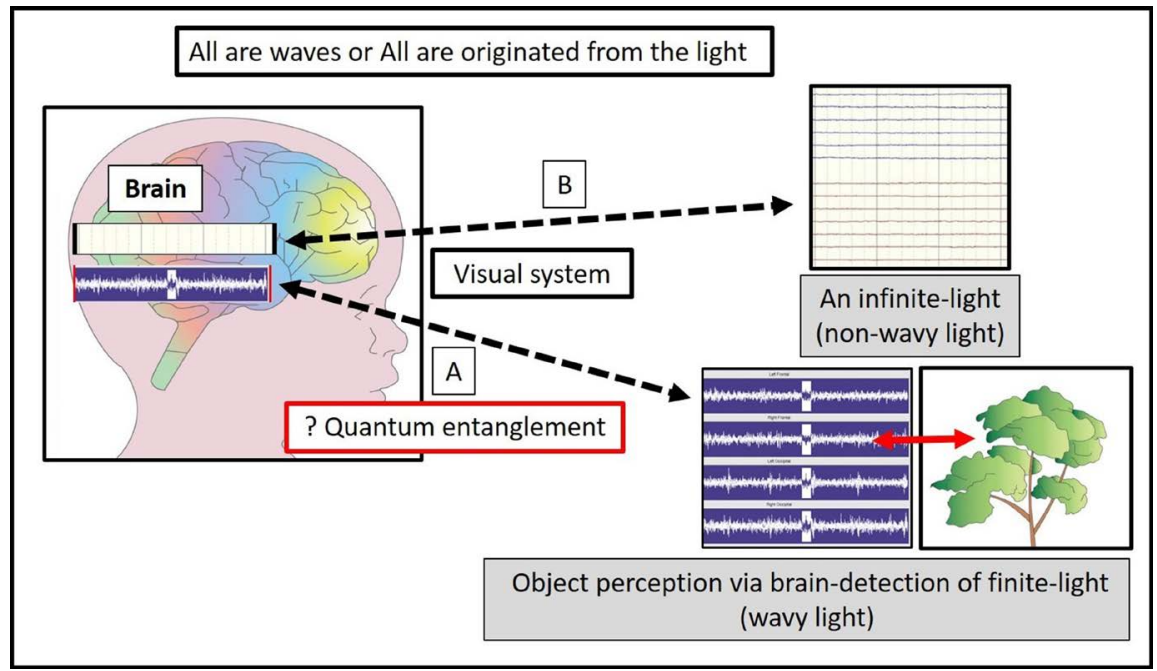

Figure 5. Human eyes (brain) can perceive the object via detection of evoked waves (A). Human brain can not perceive an infinite item such as an infinite-light because it is waveless (B). Quantum entanglement might play a role in our perception. This obviously needs quantum research to confirm the entanglement theory for perception. Note that, atom can either be particles or waves; thus a tree can also be viewed as waves or wavy item.

universe. This universe is infinite or better called it as a metaphysical infinite-universe. This infinite-universe (infinite-light) seems also to embrace our physical universe, and in reality both can be viewed as "one", that is to say the finite-light does cross the infinite-light or in other words "light upon light".

To end this discussion, I would like to pose three questions and briefly discuss one related neurotheology: 1) Can this infinite-light, which crossing the finite-light in our current physical universe be referred as dark energy? 2) Do the transitional points or areas exist between these two universes? And 3) Can those (transitional areas) be at the black holes for the greatest object such as a star and its galaxies? and thus allowing us to study it! Black hole ("dead" mechanism of a star-galaxy system) as a hypothetical transitional site between wavy and waveless items sounds interesting. Formation of a black hole would cause initial perception of brightness (emitted radiation or wavy-visible-light) and later to darkness (waveless-invisible-light) (Figure 4(D)) (Wald, 1997). However, the darkness perception of the black hole is possibly due to our limited brain perception that is to say resulted from our "low frequency wave of perception" or "finite frequency of brain perception" or "physical or eye-brain perception" or "unfree-from-body soul of perception". Once experiencing absence of physical or eye-brain perception; for instance, when becoming physically dead in the black hole, our free soul (from the bodies) might experience a frequency shift and hence reverts to the highest frequency of perception. This "infinite-frequency of perception" might appraise darkness of the black hole as the opposite! That is infinite-brightness with infinite-calmness [i.e. back to the origin/infinity]. Do look back at Figure 3 for the frequency, time and energy in two different dimensions (finite- and infinite-universe) and Figure 4 for the non-living things, living 
things and star-galaxy transitions. Finally, do note that for human body, once it is dead - no more capability to see, move, think, hear etc. It signifies soul is actually real us which has capability to see, hear, move etc (i.e. indirectly referring soul as waves or energy). In summary, non-living things (such as table, car etc.) - waves (at certain frequency) make them seen as existing or seen as solid object-like feature; and for living things (such as a cat or fish)-again waves make them seen as existing (frequency range for solid/anatomical object) and also seen as alive (respiration, brainwaves, cardiac pulses etc.-frequency range for physiological parameters). A shift in wave frequency from highest (with infinite-frequency/energy/invisible) to lower frequencies (finite-frequency) might occur for soul when it undergoes transition (Figure 4). Worthy of note here that, soul is not wave and wave is not soul-wave (energy) could merely be "an indirect manifestation" for the soul which can indeed be detected and studied.

\section{How to Prove the Existence of an Infinite-Light?}

This manuscript hypothesizes the existence of an infinite-light either at time immediately after the big bang or it was already existed prior to a big bang (as the first creation). In scientific world, theory should be followed by method. In this regard, to prove the existence of an infinite-light as a fabric for our physical universe (? unifying all theories) is not an easy task. In my opinion, one might do so when having the capability to switch the human brainwaves on and off successfully, and ask what can a person see when it is on and off (maybe in a brain-transplanted individual when his/her brainwaves become totally flat and waveless!). Another hard method, is the ability to create something faster than the ordinary photonic-light and ask a person to see at it while studying the brainwaves of visual cortex (brain). The faster it is from the speed of light, the fader it becomes to be perceived, and thus appear less wavy brainwaves at the visual cortex. In relation to this point, one may argue absence of total darkness in a dark room (or when one closes the eyes) is due to the presence of a background infinite-light which gives sense of seeing a bit of light. The infinite-light when criss-crossed by the finite-light (co-exist in the dark room) and when oxygen molecules, air or particles forming the wall inside that dark room are perceived as waves or light, a whole frequency inside the dark room is still in perceivable finite frequency range (oscillating) and hence, can be sensed by our brain or contributes a bit to "a background brainwaves" when asleep, in coma or even at rest with eyes close. Finally, one may attest a true presence of an infinite-light through stories told by most near death experience (NDE) persons who saw the light when having (near) flattened brainwaves (Drab, 1981; French, 2005). Figure 6 illustrates the methods.

\section{Conclusion}

This manuscript argues the existence of an infinite-light which explains formation of physical universe, conservation of energy, finite age of finite universe, infinite age of infinite universe, one consciousness, characters and features for the 


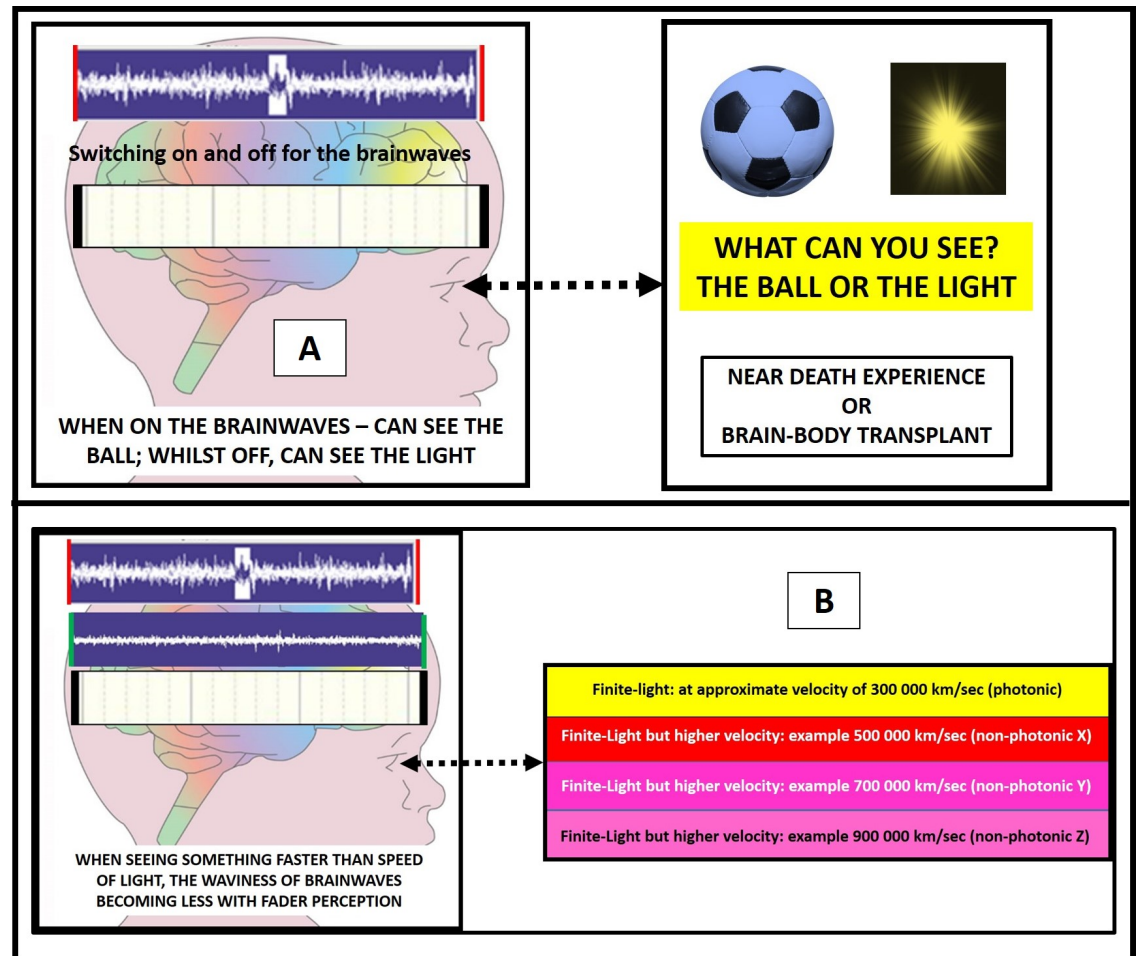

Figure 6. Illustration of experiments on how an infinite-light might be proven as in existence. These experiments are difficult and require futuristic innovations. A: By "on" the brainwaves, individual can perceive the ball; when "off", perception of light is expected. B: Brainwaves becoming less wavy when person seeing something faster than speed of light and at the same time, his or her perception becoming fader.

black hole and Olber's paradox, and steers isotropic and homogenous physical universe expansion. Its existence can be argued at time immediately after the big bang or even before the big bang (preferred). This manuscript also describes the cascade concept of light-waves-particles which can be stated as all are waves or all are originated from the light. Thus it seems that these two lights could coexist, and they may interact or criss-cross with each other. In other words: there may be "light upon light"-one is measurable and detectable via direct and indirect means; and another is yet technologically not. Finally, ones may say "the origin for the light is from the higher light".

\section{Conflicts of Interest}

The author declares no conflicts of interest regarding the publication of this paper.

\section{References}

Afshordi, N., \& Magueijo, J. (2016). Critical Geometry of a Thermal Big Bang. Physical Review D, 94, Article ID: 101301(R). https://doi.org/10.1103/PhysRevD.94.101301

Ahmed, F. A., \& Saurya, D. (2015). Cosmology from Quantum Potential. Physics Letters $B$, 741, 276-279. https://doi.org/10.1016/j.physletb.2014.12.057

Arndt, M., Nairz, O., Vos-Andreae, J., Keller, C., Van Der Zouw, G., \& Zeilinger, A. 
(1999). Wave-Particle Duality of C(60) Molecules. Nature, 401, 680-682. https://doi.org/10.1038/44348

Arp, H. C., Burbidge, G., Hoyle, F., Narlikar, J. V., \& Wickramasinghe, N. C. (1990). The Extragalactic Universe: An Alternative View. Nature, 346, 897-812. https://doi.org/10.1038/346807a0

Arthur, E. P. (2019). Quantum Cosmology and the Role of Consciousness. NeuroQuantology, 17, 104-111. https://doi.org/10.14704/nq.2019.17.1.1934

Barrow, J. D., \& Shaw, D. J. (2011). The Value of the Cosmological Constant. General Relativity and Gravitation, 43, 2555-2560. https://doi.org/10.1007/s10714-011-1199-1

Bohr, N. (1928). The Quantum Postulate and the Recent Development of Atomic Theory. Nature, 121, 580. https://doi.org/10.1038/121580a0

Cocchi, M., Gabrielli, F., Tonello, L., \& Tuszynski, J. (2017). A Dialogue on the Issue of the "Quantum Brain" between Consciousness and Unconsciousness. Journal of Integrative Neuroscience, 16, S13-S18. https://doi.org/10.3233/JIN-170062

Compton, A. H. (1923). A Quantum Theory of the Scattering of X-Rays by Light Elements. Physical Review, 21, 483-502. https://doi.org/10.1103/PhysRev.21.483

Di Biase, F. (2009). A Holoinformational Model of Consciousness. Quantum Biosystems, 3, 207-220

Meijer, D. K. F., \& Raggett, S. (2014). Quantum Physics in Consciousness Studies. The Quantum Mind Extended.

https://www.researchgate.net/profile/Dirk Meijer4/publication/275017089 Quantum Phys-

ics in Consciousness Studies The Quantum Mind Extended/links/555c62b708ae8f6 6f3ae0760/Quantum-Physics-in-Consciousness-Studies-The-Quantum-Mind-Extende d.pdf

Doesburg, S. M., Green, J. J., McDonald, J. J., \& Ward, L. M. (2009). Rhythms of Consciousness: Binocular Rivalry Reveals Large-Scale Oscillatory Network Dynamics Mediating Visual Perception. PLoS ONE, 4, e6142.

https://doi.org/10.1371/journal.pone.0006142

Drab, K. J. (1981). The Tunnel Experience: Reality or Hallucination? Anabiosis: Journal of Near-Death Studies, 1, 126-152. https://doi.org/10.17514/JNDS-1981-1-2-p126-152.

Du, X., \& Zhao, D. (2010). Scattering of Light by a System of Anisotropic Particles. Optics Letters, 35, 1518-1520. https://doi.org/10.1364/OL.35.001518

Einstein, A. (1917). Kosmologische Betrachtungen zur Allgemeinen Relativitatstheorie. In: Sitzungsberichte der Koniglich Preussischen Akademie der Wissenschaften, VI, Deutsche Akademie der Wissenschaften zu Berlin, Berlin, 142-152.

French, C. C. (2005). Near-Death Experiences in Cardiac Arrest Survivors. Progress in Brain Research, 150, 351-67. https://doi.org/10.1016/S0079-6123(05)50025-6

Georgiev, D. D., \& Glazebrook, J. F. (2018). The Quantum Physics of Synaptic Communication via the SNARE Protein Complex. Progress in Biophysics \& Molecular Biology, 135, 16-29. https://doi.org/10.1016/j.pbiomolbio.2018.01.006

Guth, A. H., \& Kaiser, D. I. (2005). Inflationary Cosmology: Exploring the Universe from the Smallest to the Largest Scales. Science, 307, 884-890.

https://doi.org/10.1126/science. 1107483

Hawking, S. W., \& Ellis, G. F. R. (1973). The Large Scale Structure of Space-Time. Cambridge: Cambridge University Press. https://doi.org/10.1017/CBO9780511524646

Hubble, E. (1927). Big Bang Theory Is Introduced. A Science Odyssey. 
https://www.pbs.org/wgbh/aso/databank/entries/dp27bi.html

Hubble, E. (1929). A Relation between Distance and Radial Velocity among Extra-Galactic Nebulae. Proceedings of the National Academy of Sciences, 15, 168-173. https://doi.org/10.1073/pnas.15.3.168

Idris, Z. (2014). Searching for the Origin through Central Nervous System: A Review and thought Which Related to Microgravity, Evolution, Big Bang Theory and Universes, Soul and Brainwaves, Greater Limbic System and Seat of the Soul. Malaysian Journal of Medical Sciences, 21, 4-11.

Idris, Z., Muzaimi, M., Ghani, R., Idris, B., Kandasamy, R., \& Abdullah, J. (2014). Principles, Anatomical Origin and Applications of Brainwaves: A Review, Our Experience and Hypothesis Related to Microgravity and the Question on Soul. Journal of Biomedical Science and Engineering, 7, 435-445. https://doi.org/10.4236/jbise.2014.78046

Keppler, J. (2018). The Role of the Brain in Conscious Processes: A New Way of Looking at the Neural Correlates of Consciousness. Frontiers in Psychology, 9, 1346. https://doi.org/10.3389/fpsyg.2018.01346

Meijer, D. K. F., \& Geesink, H. (2019). Life and Consciousness Are Guided by a Semi-Harmonic EM Background Field. NeuroQuantology, 17, 37-44.

https://doi.org/10.14704/nq.2019.17.4.2074

Poe, E. A. (1848). Eureka: A Prose Poem. http://xroads.virginia.edu/ hyper/poe/eureka.html

Ratcliffe, H. (2010). Anomalous Redshift Data and the Myth of Cosmological Distance. Journal of Cosmology, 4, 693-718.

Sakai, K. (2018). Simultaneous Measurement of Wave and Particle Properties Using Modified Young's Double-Slit Experiment. Journal for Foundations and Applications of Physics, 5, 49-54.

Shi, L., Galvez, E. J., \& Alfano, R. R. (2016). Photon Entanglement through Brain Tissue. Scientific Reports, 6, Article No. 37714. https://doi.org/10.1038/srep37714

Subhash, K. (2009). The Universe, Quantum Physics, and Consciousness. Journal of Cosmology, 3, 500-510.

Thomson, G. P. (1927). Diffraction of Cathode Rays by a Thin Film. Nature, 119, 890. https://doi.org/10.1038/119890a0

Van Flandern, T. C. (2002). The Top 30 Problems with the Big Bang. Meta Research Bulletin, 11, 6-13.

Vilenkin, A. (1983). The Birth of Inflationary Universes. Physical Review D, 27, 2848. https://doi.org/10.1103/PhysRevD.27.2848

Wald, R. M. (1997). Gravitational Collapse and Cosmic Censorship. In B. R. Iyer, \& B. Bhawal (Eds.), Black Holes, Gravitational Radiation and the Universe (pp. 69-86). Berlin: Springer. https://doi.org/10.1007/978-94-017-0934-7 5

Yuan, Y. et al. (2018). Experimental Demonstration of Wave-Particle Duality Relation Based on Coherence Measure. Optics Express, 26, 4470-4478. https://doi.org/10.1364/OE.26.004470

Zarkeshian, P., Kumar, S., Tuszynski, J., Barclay, P., \& Simon, C. (2018). Are There Optical Communication Channels in the Brain? Frontiers in Bioscience, 23, 1407-1421. https://doi.org/10.2741/4652 